\title{
Access to medicines for rare diseases: beating the drum for primary ciliary dyskinesia
}

\section{Suzanne Crowley (10) ${ }^{1}$, Inês Azevedo ${ }^{2,3}$, Mieke Boon $^{4}$, Andrew Bush ${ }^{5}$, Ernst Eber ${ }^{6}$, Eric Haarman ${ }^{7}$, Bulent Karadag $^{8}$, Karsten Kötz ${ }^{9}$, Margaret Leigh ${ }^{10}$, Antonio Moreno-Galdó ${ }^{11,12}$, Huda Mussaffi ${ }^{13}$, Kim G. Nielsen (1) ${ }^{14}$, Heymut Omran ${ }^{15}$, Jean-François Papon ${ }^{16}$, Petr Pohunek (10 ${ }^{17}$, Kostas Priftis ${ }^{18}$, Bernhard Rindlisbacher ${ }^{19}$, Francesca Santamaria ${ }^{20}$, Arunas Valiulis (1) ${ }^{21,22}$, Michal Witt ${ }^{23}$, Panayiotis Yiallouros ${ }^{24}$, Zorica Zivkovic ${ }^{25,26}$, Claudia E. Kuehni (1) ${ }^{27}$ and Jane S. Lucas ${ }^{28}$ for BEAT-PCD}

\begin{abstract}
${ }^{1}$ Paediatric Dept for Lung and Allergic diseases, Oslo University Hospital, Oslo, Norway. ${ }^{2}$ Centro MaternoPediátrico, Centro Hospitalar Universitário de S. João, Porto, Portugal. ${ }^{3}$ Departamento de GinecologiaObstetrícia e Pediatria, Faculdade de Medicina, Universidade do Porto, Porto, Portugal. ${ }^{4}$ Dept of Paediatrics, University Hospital Gasthuisberg, Leuven, Belgium. ${ }^{5}$ Depts of Paediatrics and Paediatric Respiratory Medicine, Imperial College and Royal Brompton Hospital, London, UK. ${ }^{6}$ Division of Paediatric Pulmonology and Allergology, Dept of Paediatrics and Adolescent Medicine, Medical University of Graz, Graz, Austria. ${ }^{7}$ Dept of Pediatric Pulmonology, VU University Medical Center, Amsterdam, The Netherlands. ${ }^{8}$ Dept of Pediatric Pulmonology, Marmara University, School of Medicine, Istanbul, Turkey. ${ }^{9}$ Queen Silvias Children's Hospital, Sahlgrenska University Hospital, Gothenburg, Sweden. ${ }^{10}$ Dept of Pediatrics and Marsico Lung Institute, University of North Carolina at Chapel Hill, Chapel Hill, NC, USA. ${ }^{11}$ Pediatric Pulmonology Section, Hospital Universitari Vall d'Hebron, Barcelona, Spain. ${ }^{12}$ Universitat Autònoma de Barcelona, CIBERER, Barcelona, Spain. ${ }^{13}$ Schneider Children's Medical Center of Israel, Petach-Tikva and Sackler School of Medicine, Tel Aviv University, Tel Aviv, Israel. ${ }^{14}$ Danish PCD Centre, Pediatric Pulmonary Service, Dept of Pediatrics and Adolescent Medicine, Rigshospitalet (Copenhagen University Hospital), Copenhagen, Denmark. ${ }^{15}$ Dept of General Pediatrics, University Hospital, Westfalian Wilhelms-University, Muenster, Germany. ${ }^{16}$ AP-HP, Hôpital Kremlin-Bicetre, Service d'ORL et de Chirurgie Cervico-Faciale and Faculté de Médecine, Université ParisSaclay, 94070 Le Kremlin-Bicêtre, INSERM, U955 and CNRS, ERL 7240, Créteil, France. ${ }^{17}$ Paediatric Dept, Second Faculty of Medicine, Charles University and Motol University Hospital, Prague, Czech Republic. ${ }^{18} 3$ rd Dept of Paediatrics, University General Hospital Attikon, National and Kapodistrian University of Athens, Athens, Greece. ${ }^{19}$ Patient Association Kartagener Syndrom und Primäre Ciliäre Dyskinesie e.V., Steffisburg, Switzerland. ${ }^{20}$ Pediatric Pulmonology, Dept of Translational Medical Sciences, Federico II University, Azienda Ospedaliera Universitaria Federico II, Naples, Italy. ${ }^{21}$ Vilnius University Medical Faculty, Institute of Clinical Medicine, Clinic of Children's Diseases, Vilnius, Lithuania. ${ }^{22}$ European Academy of Paediatrics (EAP/UEMSSP), Brussels, Belgium. ${ }^{23}$ Dept of Molecular and Clinical Genetics, Institute of Human Genetics Polish Academy of Sciences, Poznan, Poland. ${ }^{24}$ Medical School, University of Cyprus, Nicosia, Cyprus. ${ }^{25}$ Children's Hospital for Lung Diseases and TB, Medical Centre "Dr Dragisa Misovic", Belgrade, Serbia. ${ }^{26}$ Faculty of Pharmacy Novi Sad, Business Academy, Novi Sad, Serbia. ${ }^{27}$ Institute of Social and Preventive Medicine and Paediatric Respiratory Medicine, Children's University Hospital of Bern, University of Bern, Bern, Switzerland. ${ }^{28}$ Primary Ciliary Dyskinesia Centre, University Hospital Southampton NHS Foundation Trust and Clinical and Experimental Medicine, University of Southampton, Southampton, UK.
\end{abstract}

Correspondence: Suzanne Crowley, Paediatric Dept for Lung and Allergic diseases, Oslo University Hospital, Sognvannsveien 20, 0027 Oslo, Norway. E-mail: suzanne.crowleylagmail.com

\section{@ERSpublications}

Primary ciliary dyskinesia, a rare disease causing bronchiectasis, lacks a sound evidence base for treatment. @beatpcd proposes 1) forming a PCD European clinical trial network to address this situation and 2) conducting n-of-1 trials to access medication. https://bit.ly/3j5blfM

Cite this article as: Crowley S, Azevedo I, Boon $\mathrm{M}$ et al. Access to medicines for rare diseases: beating the drum for primary ciliary dyskinesia. ERJ Open Res 2020; 6: 00377-2020 [https:/doi.org/ $10.1183 / 23120541.00377-2020]$. 
Rare diseases are collectively common, affecting an estimated $6.2 \%$ of the world's population [1], but each rare disease affects fewer than 4 to 5 in 10000 individuals in Europe or less than 200000 individuals in the USA [2]. Patients with rare diseases are often disadvantaged by late diagnosis and off-label prescribing of medicines [3]. Primary ciliary dyskinesia (PCD) is a genetic disease of impaired motile ciliary function that does not have a unique International Classification of Diseases (ICD)-10 code or licensed treatments, although Q34.8 denoting “other specified malformations of the respiratory tract" including nasopharyngeal atresia has also been applicable to PCD since 2017. The disease is characterised by mucus stagnation leading to chronic airway infection, bronchiectasis, chronic rhinosinusitis, reduced fertility and abnormalities of organ laterality with an associated increased risk of complex congenital heart disease [4]. The estimated prevalence of PCD in Europe is around 1 in 10000 to 1 in 20000 [5]. The international PCD cohort (iPCD) includes over 3800 PCD patients ranging in age from under 12 months to over 80 years, from Europe, Northern and Southern America, Australia and Western Asia [6]. Under-diagnosis of PCD is due to a lack of awareness among the general public and physicians in general, as well as a lack of diagnostic expertise in some countries [7]. Tools to help physicians identify patients needing testing (e.g. PICADAR) [8] and the European Respiratory Society (ERS) guidelines for diagnostic testing [9] aim to improve this. In contrast to cystic fibrosis (CF), a monogenic disease, PCD is caused by mutations in one of at least 45 identified genes for which there is no effective mutation-specific therapy; this is likely to be a long way off for most patients [10]. Thus, treatment aims to prevent and manage disease complications. Even then, the lack of an evidence base for supportive treatment in PCD means that treatment recommendations are based on expert opinion and extrapolated from CF despite differing pathophysiology [11].

There are only two published double-blind, randomised, controlled treatment studies in PCD, each a milestone achievement given recruitment difficulties $[12,13]$. Paucity of evidence means that patients are exposed to risks including inappropriate dosing [14], deleterious side-effects [15] and increased treatment burden with ineffective therapies. A further consequence has been varying reimbursement policies for medicines, restricting access to inhaled antibiotics for example, with some countries resorting to cheaper alternatives with an inferior safety profile [16] and others displaying regional policy differences [17]. This restriction of access to medicines and the difficulty in conducting clinical trials in PCD is particularly concerning in regard to children. In this editorial, we show how PCD patients are being discriminated against by inequalities of access to medications and propose two solutions. The long-term solution is conducting more randomised controlled trials (RCTs) by the formation of a clinical trials network similar to the existing European and American CF Clinical Trials Networks (ECFS-CTN, CFF-CTN, respectively). Further, the empowerment of and engagement with patient organisations is fundamental to patient recruitment for clinical trials and should be prioritised. The second solution, which can end this discrimination by being implemented immediately, is to allow the prescription of inhaled medications to PCD patients if benefit is demonstrated in an appropriate n-of- 1 trial.

\section{Treatment evidence}

PCD lung disease is not trivial. Lung function is below $80 \%$ predicted in one-third of children at diagnosis [18] and is impaired to a similar degree in childhood compared with CF [19]. A third of paediatric and adult patients will lose more than 10 percentage points of lung function over a 10-year period [18]. Data from the American bronchiectasis registry show that adults with PCD have worse lung function, greater morbidity and are more likely to be infected with Pseudomonas aeruginosa than adults with idiopathic bronchiectasis, common variable immunodeficiency and alpha-1 antitrypsin deficiency [20]. Mobilisation of airway secretions is the cornerstone of PCD management [11]. Airway mucus in PCD and CF share similar biophysical properties [21]. The first ever prospective controlled trial of treatment in PCD, a randomised, double-blind cross-over trial of 3 months' treatment with nebulised $7 \%$ hypertonic saline (HS) and $0.9 \%$ saline, measured quality of life (QoL) and lung function in 22 adults with PCD, finding a significant difference favouring HS only in perception of health [12]. The small number of patients, a result of recruitment difficulty, meant that the study wasn't sufficiently powered to detect differences in the primary outcome measures. An unintended consequence of these negative findings has been the refusal of some insurance companies in the USA to fund HS and nebulisers for PCD patients (M. Leigh, personal communication); payment for HS is not reimbursed in several European countries, a situation remedied by some hospital pharmacies producing their own HS.

Compared with placebo, treatment with inhaled recombinant human deoxyribonuclease (rhDNase) for longer than 6 months is associated with a decrease in pulmonary exacerbations and an improvement in lung function in CF patients [22]. There have been anecdotal reports of improved sputum expectoration and lung function after short- and longer-term treatment with rhDNase in four children with PCD who were deteriorating [23-25]. Conversely, studies of rhDNase in adults with non-CF bronchiectasis showed no clinical benefits in one study [26] and increased frequency of pulmonary exacerbations with worsened 
lung function in another [15]. While rhDNase on an individual trial basis can be used when lung function is deteriorating despite maximal medical therapy [27], the prohibitive cost precludes its use in most European countries where the patient must pay. Looking to the future, a more affordable rhDNase (biosimilar JHL 1922) has undergone a phase 1 clinical trial in the Netherlands [28]; a phase 3 trial in CF patients is planned. Such clinical research initiatives represent an opportunity that should be actively pursued by the PCD community.

PCD lung disease is characterised by repeated pulmonary exacerbations. The newly published, multicentre, double-blind, randomised placebo-controlled study, BESTCILIA, showed that thrice weekly maintenance treatment with azithromycin for 6 months in 90 children and adults halved the rate of respiratory exacerbations and significantly reduced the rate of sputum carriage of pathogenic bacteria compared with placebo [13]. This milestone study confirms and extends to PCD data from studies in CF and bronchiectasis, and paves the way for the development of precision medicine approaches for PCD to determine who would most benefit from this treatment [29]. Furthermore, P. aeruginosa infection in PCD is common; infection rates as high as $37 \%$ have been reported in children [30], and in $39 \%$ and $51 \%$ of adolescents and adults, respectively [31]. This compares, in the latter two age groups, with $12 \%$ and $26 \%$ for bronchiectasis and 60\% and 67\% for CF [31]. Data from the American adult bronchiectasis registry showed a $63.5 \%$ prevalence of at least one positive $P$. aeruginosa sputum culture in the previous year [20]. Chronic $P$. aeruginosa infection in PCD is also associated with poorer lung function, especially in women [32], while the impact on life expectancy is unknown. Prompt $P$. aeruginosa eradication is recommended in PCD, but with no evidence to support dosing strategies or duration of treatment [11, 27]. A survey of eradication strategies in European PCD centres showed that in some countries (Belgium, Bulgaria, Turkey and Ukraine), inhaled anti- $P$. aeruginosa antibiotics are not generally prescribed due to lack of reimbursement of costs [16], leaving the patient with either the risk of becoming chronically infected or having to pay, which is prohibitively expensive for most, or being falsely reclassified as having CF.

\section{Proposal}

CF is the only chronic suppurative lung disease for which treatment with inhaled antibiotics and rhDNase has been approved by the European Medicines Agency and the US Food and Drug Administration. The five key criteria for reimbursement of medicines in countries within Europe are demonstration of therapeutic benefit, medical necessity, safety, cost-effectiveness and budget impact [33]. Further, reimbursement may be product-specific, disease-specific, population groups-specific (e.g. children or pensioners), or consumption based. Demonstration of fulfilment of these five criteria is currently impossible for any inhaled PCD treatments. Given that a cure for PCD is unlikely in the foreseeable future, efforts also need to be directed towards establishing the efficacy in PCD of medicines shown to be effective in treating other causes of bronchiectasis. The BESTCILIA azithromycin study represents an important first step towards this goal and demonstrates the ability of the PCD community to come together to perform RCTs. A formal clinical trials network might be along the lines of the European CF Society Clinical Trial Network (ECFS-CTN) and the CF Foundation Clinical Trial Network (CFF-CTN), ideally involving every diagnosed patient. As soon as one study is completed and the results analysed, the next study should begin. This method, of including virtually every patient in a clinical study, has been adopted for decades in rare childhood cancers, where it has led to impressive improvements in long-term outcomes [34]. BEAT-PCD has recently been granted an ERS Clinical Research Collaboration to advance clinical and translational research in PCD and setting the framework for the formation of a PCD-CTN would fall within this remit. However, clinical trials take time and while they are the gold-standard, it is unreasonable that patients should be denied treatment pending the outcome of such trials. We urge regulatory authorities to fund inhaled medications if they show benefit in the individual patient in an appropriately conducted, n-of- 1 trial. It is simply not good enough to refuse to fund because "there is no evidence". Absence of evidence should not be interpreted as absence of benefit. Even within RCTs, there is usually a wide range of individual responses, and in clinical practice, an individualised approach is taken when planning therapy. In summary, we, as a group of experts managing the care of PCD patients in Europe and the USA, wish to highlight the discrimination experienced by PCD patients, the likely detrimental effects on exacerbation frequency, future lung function, QoL and healthcare costs, and propose a solution which can be implemented now while on the road to conducting more RCTs.

Conflict of interest: S. Crowley has nothing to disclose. I. Azevedo has nothing to disclose. M. Boon reports grants from Horizon 2020 MyCyFAPP and KOOR post-doctoral funding from University Hospital Leuven, outside the submitted work. A. Bush has nothing to disclose. E. Eber has nothing to disclose. E. Haarman has nothing to disclose. B. Karadag has nothing to disclose. K. Kötz has nothing to disclose. M. Leigh reports a primary ciliary dyskinesia (PCD) research grant from the National Institutes of Health and a grant for a PCD clinical trial from Parion Sciences, outside the submitted work. A. Moreno-Galdó reports support for travel to and registration for medical conferences from Actelion, Abbvie and Novartis, outside the submitted work. H. Mussaffi has nothing to disclose. K.G. Nielsen has nothing to disclose. H. Omran has nothing to disclose J-F. Papon has nothing to disclose. P. Pohunek reports grants from the 
Ministry of Health, Czech Republic, during the conduct of the study. K. Priftis has nothing to disclose. B. Rindlisbacher has nothing to disclose. F. Santamaria has nothing to disclose. A. Valiulis has nothing to disclose. M. Witt has nothing to disclose. P. Yiallouros has nothing to disclose. Z. Zivkovic has nothing to disclose C.E. Kuehni reports grants from Swiss National Science Foundation during the conduct of the study. J.S. Lucas has nothing to disclose.

Support statement: The authors are national representatives participating in the European Respiratory Society-sponsored clinical research collaboration (2020) and European Union-sponsored Cooperating in Science and Technology action BEAT-PCD: Better Experimental Approaches to Treat Primary Ciliary Dyskinesia (BM1407, 2014-2019). BEAT-PCD is a network of scientists and clinicians coordinating research from basic science through to clinical care with the intention of developing treatments and diagnostics that lead to improved long-term outcomes for patients. M. Boon, E. Haarman, A. Moreno-Galdó, K.G. Nielsen, H. Omran, J-F. Papon, P. Pohunek, B. Rindlisbacher, F. Santamaria, P. Yiallouros and J.S. Lucas are core members of the European Reference Network on rare lung diseases, ERN-LUNG, approved on 15 December 2016 by the European Commission's Board of Member States. ERN-LUNG is dedicated to ensuring and promoting excellence in care and research for the benefit of patients affected by rare respiratory diseases, and is committed Europe-wide and globally to the prevention, diagnosis and treatment of rare respiratory diseases through patient care and advocacy, education and research. C.E. Kuehni receives support from the Swiss National Science Foundation (SNF 320030B_192804). M. Leigh is a MC Observer in BEAT-PCD and receives support from the Genetic Disorders of Mucociliary Clearance Consortium (US National Institutes of Health grant U54HL096458; principal investigators: S.D. Davis and T. Ferkol), a part of the NCATS Rare Diseases Clinical Research Network (RDCRN) that is funded through a collaboration between NCATS and NHLBI. K.G. Nielsen is supported by the Children's Lung Foundation (Denmark). M. Boon is supported by a post-doctoral KOOR grant (University Hospital Leuven).

\section{References}

$1 \quad$ Ferreira CR. The burden of rare diseases. Am J Med Genet 2019; 179A: 885-892.

2 Richter T, Nestler-Parr S, Babela R, et al. Rare disease terminology and definitions-a systematic global review: report of the ISPOR Rare Disease Special Interest Group. Value Health 2015; 18: 906-914.

3 Tadrous M, Khuu W, Paterson JM, et al. Off-label use of inhaled tobramycin in Ontario, Canada. Thorax 2016; 71: 862-864.

4 Goutaki M, Meier AB, Halbeisen FS, et al. Clinical manifestations in primary ciliary dyskinesia: systematic review and meta-analysis. Eur Respir J 2016; 48: 1081-1095.

5 Kuehni CE, Frischer T, Strippoli M-PF, et al. Factors influencing the age at diagnosis of primary ciliary dyskinesia in European children. Eur Respir J 2010; 36: 1248-1258.

6 Goutaki M, Maurer E, Halbeisen FS, et al. The international primary ciliary dyskinesia cohort (iPCD Cohort): methods and first results. Eur Respir J 2017; 49: 1601181.

7 Behan L, Galvin AD, Rubbo B, et al. Diagnosing primary ciliary dyskinesia: an international patient perspective. Eur Respir J 2016; 48: 1096-1107.

8 Behan L, Dimitrov BD, Kuehni CE, et al. PICADAR: a diagnostic predictive tool for primary ciliary dyskinesia. Eur Respir J 2016; 47: 1103-1112.

9 Lucas JS, Barbato A, Collins SA, et al. European Respiratory Society guidelines for the diagnosis of primary ciliary dyskinesia. Eur Respir J 2017; 49: 1601090.

10 Lucas JS, Davis SD, Omran H, et al. Primary ciliary dyskinesia in the genomics age. Lancet Respir Med 2020; 8 : 202-216.

11 Lucas JS, Alanin MC, Collins S, et al. Clinical care of children with primary ciliary dyskinesia. Expert Rev Respir Med 2017; 11: 779-790.

12 Paff T, Daniels JM, Weersink EJ, et al. A randomised controlled trial on the effect of inhaled hypertonic saline on quality of life in primary ciliary dyskinesia. Eur Respir J 2017; 49: 1601770.

13 Kobbermagel HE, Buchvald FF, Haarman EG, et al. Efficacy and safety of azithromycin maintenance therapy in primary ciliary dyskinesia (BESTCILIA): a multicenter, double-blind, randomised, placebo-controlled, phase 3 trial. Lancet Respir Med 2020; 8: 493-505.

14 Higgins KL, Noda C, Stultz JS. Extended interval tobramycin pharmacokinetics in a pediatric patient with primary ciliary dyskinesia presenting with an acute respiratory exacerbation. J Pediatr Pharmacol Ther 2018; 23: 159-163.

15 O'Donnell AE, Barker AF, Ilowite JS, et al. Treatment of idiopathic bronchiectasis with aerosolized recombinant human DNase I. rhDNase Study Group. Chest 1998; 113: 1329-1334.

16 Crowley S, Holgersen MG, Nielsen KG. Variation in treatment strategies for the eradication of Pseudomonas aeruginosa in primary ciliary dyskinesia across European centers. Chron Respir Dis 2019; 16: 1479972318787919.

17 Czech M, Baran-Kooiker A, Atikeler K, et al. A review of rare disease policies and orphan drug reimbursement systems in 12 Eurasian countries. Front Public Health 2019; 7: 416.

18 Marthin JK, Petersen N, Skovgaard LT, et al. Lung function in patients with primary ciliary dyskinesia: a cross-sectional and 3-decade longitudinal study. Am J Respir Crit Care Med 2010; 181: 1262-1268.

19 Halbeisen FS, Goutaki M, Spycher BD, et al. Lung function in patients with primary ciliary dyskinesia: an iPCD Cohort study. Eur Respir J 2018; 52: 1801040

20 Eden E, Choate R, Barker A, et al. the clinical features of bronchiectasis associated with alpha-1 antitrypsin deficiency, common variable immunodeficiency and primary ciliary dyskinesia-Results from the U.S Bronchiectasis Research Registry. Chronic Obstr Pulm Dis 2019; 6: 145-153.

21 Bush A, Payne D, Pike S, et al. Mucus properties in children with primary ciliary dyskinesia: comparison with cystic fibrosis. Chest 2006; 129: 118-123.

22 Yang C, Montgomery M. Dornase alfa for cystic fibrosis. Cochrane Database Syst Rev 2018; 9: CD001127.

23 Desai M, Weller PH, Spencer DA. Clinical benefit from nebulized recombinant DNase in Kartagener's syndrome. Pediatr Pulmonol 1995; 20: 307-308.

24 ten Berge M, Brinkhorst G, Kroon AA, et al. DNase treatment in primary ciliary dyskinesia: assessment by nocturnal pulse oximetry. Pediatr Pulmonol 1999; 27: 59-61.

25 El-Abiad NM, Clifton S, Nasr SZ. Long-term use of nebulized human recombinant DNase1 in two siblings with primary ciliary dyskinesia. Respir Med 2007; 101: 2224-2226. 
Wilkinson M, Sugumar K, Milan SJ, et al. Mucolytics for bronchiectasis. Cochrane Database Syst Rev 2014; 5 CD001289.

27 Shapiro AJ, Zariwala MA, Ferkol T, et al. Diagnosis, monitoring, and treatment of primary ciliary dyskinesia: PCD foundation consensus recommendations based on state of the art review. Pediatr Pulmonol 2016; 51: $115-132$.

28 JHL Biotech, Inc. A Comparative Safety and Pharmacokinetic Study of JHL1922 and Pulmozyme in Healthy Subjects. ClinicalTrials.gov Identifier: NCT03586076. Date last updated: 7 January 2020. https://clinicaltrials.gov/ ct2/show/NCT03586076

29 Poeta M, Borelli M, Santamaria F. Azithromycin for primary ciliary dyskinesia: a milestone. Lancet Respir Med 2020; 8: 429-430.

30 Maglione M, Bush A, Nielsen KG, et al. Multicenter analysis of body mass index, lung function, and sputum microbiology in primary ciliary dyskinesia. Pediatr Pulmonol 2014; 49: 1243-1250.

31 Chang H, Adjemian J, Dell SDM, et al. Similarities between CF and PCD: Airway pathogens and lung function Prevalence of airway microbial flora in primary ciliary dyskinesia. Am J Respir Crit Care Med 2015; 191 : A1798.

32 Frija-Masson J, Bassinet L, Honoré I, et al. Clinical characteristics, functional respiratory decline and follow-up in adult patients with primary ciliary dyskinesia. Thorax 2017; 72: 154-160.

33 World Health Organization (WHO regional office for Europe). Medicines reimbursement policies in Europe, 2018. https://www.euro.who.int/_data/assets/pdf_file/0011/376625/pharmaceutical-reimbursement-eng.pdf Accessed 21 May 2020.

34 Kuehni CE, Goutaki M, Kobbernagel HE. Hypertonic saline in patients with primary ciliary dyskinesia: on the road to evidence-based treatment for a rare lung disease. Eur Respir J 2017; 49: 1602514. 